\title{
Liquid crystal hyperbolic metamaterial for wide-angle negative-positive refraction and reflection
}

\author{
G. Pawlik, ${ }^{1, *}$ K. Tarnowski, ${ }^{1}$ W. Walasik, ${ }^{2,3}$ A. C. Mitus, ${ }^{1}$ and I. C. Khoo ${ }^{4}$ \\ ${ }^{1}$ Institute of Physics, Wroclaw University of Technology, Wybrzeze Wyspianskiego 27, 50-370 Wroclaw, Poland \\ ${ }^{2}$ Aix Marseille Universite, CNRS, Centrale Marseille, Institut Fresnel, UMR 7249, 13013 Marseille, France \\ ${ }^{3}$ Institut de Ciencies Fotoniques, Universitat Politecnica de Catalunya, Castelldefels, Barcelona 08860, Spain \\ ${ }^{4}$ Department of Electrical Engineering, Pennsylvania State University, University Park, Pennsylvania 16802, USA \\ *Corresponding author: Grzegorz.Pawlik@pwr.wroc.pl
}

Received January 6, 2014; revised February 18, 2014; accepted February 18, 2014; posted February 18, 2014 (Doc. ID 204086); published March 19, 2014

\begin{abstract}
We show that nanosphere dispersed liquid crystal (NDLC) metamaterial can be characterized in near IR spectral region as an indefinite medium whose real parts of effective ordinary and extraordinary permittivities are opposite in signs. Based on this fact we designed an electro-optic effect: an external electric-field-driven switch between normal refraction, negative refraction, and reflection of TM incident electromagnetic wave from the boundary vacuum/NDLC. A detailed analysis of its functionality is given based on effective medium theory combined with a study of negative refraction in anisotropic metamaterials and finite elements simulations. (c) 2014 Optical Society of America

OCIS codes: (230.3720) Liquid-crystal devices; (160.3918) Metamaterials; (230.2090) Electro-optical devices. http://dx.doi.org/10.1364/OL.39.001744
\end{abstract}

Novel metamaterials [1,2], in particular those for which the spatial distribution of optical parameters can be specifically tailor made, have proven to be a viable route to realize various linear, nonlinear, or tunable optical properties and processes. Amongst them, the hyperbolic metamaterials (HMs) - indefinite metamaterials, for which the permittivity and permeability tensors are negative along only certain of the principal axes of the metamaterial [3] - have attracted intense interest because of possibilities of optical steering and manipulation. Closely related are optical effects, such as negative refraction [ㄴ,5], hyperlensing [6-9], cancellation of reflection, and transmission [10]. Optical devices based on indefinite media have been designed including for example, polarization beam splitters [11], angular filters [12], and optical transmission modulators [13]. Theoretical approaches to handle the negative refraction in anisotropic indefinite media have also been presented $[4,14,15]$.

Tunability and fabrication simplicity are important requirements in metamaterial development. One of the few metamaterials that offer tunability [16] is nematic liquid crystal (NLC) doped with coated core-shell spheres (NDLC) [17]. Recently we have demonstrated a negative refraction in near IR in this system with a planar NLC configuration for a wide interval of incident angles of electromagnetic (EM) wave, using finite elements (FE) calculations (Comsol) $[18,19]$. Similar results in visible light range were presented in [20], where it was shown that a HM consisting of NLC doped with a sufficiently high concentration of silver nanoparticles can become a HM; all-angle negative refraction for homeotropic NLC orientation was demonstrated based on geometric arguments.

The point we would like to emphasize here is that whether a medium is indefinite is neither necessary $[21,22]$ nor a sufficient condition for negative refraction; we shall show below that for a HM a positive refraction as well as reflection can take place. Thus, additional quantitative calculations are necessary to trace the path of EM in an indefinite medium.
Described in this Letter is the design, using effective medium approximation and FE calculations, an electric-field-driven device for switching in NDLC HM between three scenarios: negative refraction, positive refraction, and reflection for wide intervals of angles of incidence of EM and of NLC director orientations. To this end, the approach based on negative refraction in anisotropic metamaterials $[14,15]$ is used.

Consider a rectangular NLC cell with thickness $L$ along $x$ direction, filled with NDLC metamaterial described below, with planar alignment of NLC molecules in $x-y$ plane [Fig. 1(a)]. The incident light with transverse magnetic (TM) polarization $(\vec{E}$ in $x-y$ plane and $\vec{B}$ along $z$ direction) propagates in $x-y$ plane and impinges as an extraordinary wave onto the NLC host. The permittivity tensor for NLC reads [23]:

$$
\varepsilon_{\mathrm{LC}}=\left(\begin{array}{ccc}
\varepsilon_{\perp}+\Delta \varepsilon \cos ^{2} \gamma & \Delta \varepsilon \sin \gamma \cos \gamma & 0 \\
\Delta \varepsilon \sin \gamma \cos \gamma & \varepsilon_{\perp}+\Delta \varepsilon \sin ^{2} \gamma & 0 \\
0 & 0 & \varepsilon_{\perp}
\end{array}\right),
$$

(a)

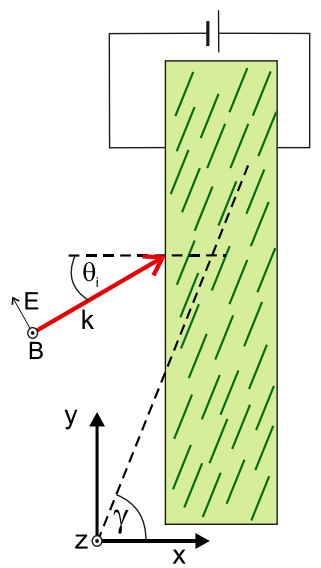

(b)

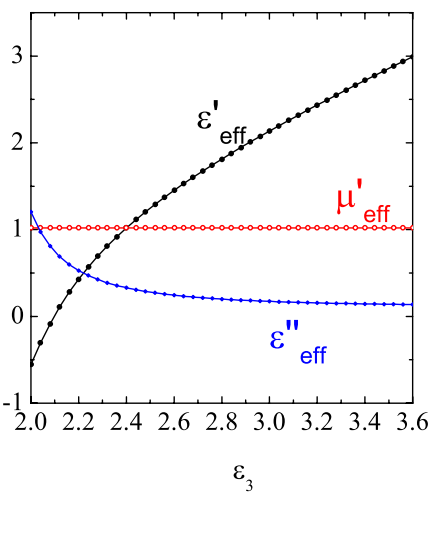

Fig. 1. (a) Geometry of the system. (b) Dependence of effective permittivity and permeability on permittivity of the LC host $\varepsilon_{3}$, Eq. (2). 
where $\gamma$ denotes the angle between the $+x$ axis and the director $\vec{n}, \Delta \varepsilon=\varepsilon_{\|}-\varepsilon_{\perp}, \varepsilon_{\|}=n_{e}^{2}, \varepsilon_{\perp}=n_{o}^{2}$.

The NDLC metamaterial [17] comprises the host NLC containing uniformly distributed nonmagnetic spheres with core radius $r_{1}$, made of polaritonic material and semiconductor shell (Drude material) with thickness $d$. The effective permittivity and permeability of NDLC were calculated in [17] using Mie theory and the Maxwell Garnet mixing rule:

$$
\varepsilon_{\mathrm{eff}}=\varepsilon_{3}\left(\frac{k_{3}^{3}+4 \pi i N a_{1}}{k_{3}^{3}-2 \pi i N a_{1}}\right), \quad \mu_{\mathrm{eff}}=\frac{k_{3}^{3}+4 \pi i N b_{1}}{k_{3}^{3}-2 \pi i N b_{1}},
$$

where $k_{3}=\sqrt{\varepsilon_{3}} 2 \pi / \lambda, \lambda$ denotes the free-space wavelength, $N$ is the number density of the spheres, and $a_{1}$, $b_{1}$ are the scattering coefficients. $\varepsilon_{3}$ denotes the permittivity of the NLC host along the main axes. This interpretation, put forward in [17], accounts for the anisotropy of the medium by treating it as isotropic and homogeneous along the two main axes. The effective permittivity tensor components in their own axes are then calculated from Eq. (2).

Parameters used in this Letter are $r_{1}=0.05 \mu \mathrm{m}$, $d=0.01 \mu \mathrm{m}, f=0.06, \varepsilon(\infty)=17, \omega_{T} / 2 \pi=360 \mathrm{THz}$, $\omega_{L} / 2 \pi=855 \mathrm{THz}, \gamma_{1} / 2 \pi=3.75 \mathrm{THz}, \omega_{p} / 2 \pi=230 \mathrm{THz}$, $\gamma_{2}=\omega_{p} / 60$, and $\lambda=1.75 \mu \mathrm{m}$; see Ref. [1]] for the definitions. The material parameters of the core (shell) are those from Ref. [17] scaled by a factor 1.5 (1.7). Equation (2) is valid in long-wavelength limit $\Lambda \equiv \lambda /\left(r_{1}+\right.$ d) $\gg 1$ (dipolar approximation) and for low values of filling factor $f=4 \pi\left(r_{1}+d\right)^{3} N / 3$ (mixing rule). In our case $\Lambda \approx 30, f=0.06$, which justifies the applicability of the approach based on Eq. (2). Plots of the real parts $\varepsilon_{\text {eff }}^{\prime}=\Re\left\{\varepsilon_{\text {eff }}\right\}, \mu_{\text {eff }}^{\prime}=\Re\left\{\mu_{\text {eff }}\right\}$ in function of $\varepsilon_{3}$ are shown in Fig. 1(b).

The propagation of a TM incident wave in this medium can be described by specifying $\varepsilon_{\text {eff }}^{\prime}$ in the propagation plane along principal axes and $\left(\mu_{\mathrm{eff}}^{\prime}\right)_{z z}$ [4]. Those components were calculated from Eq. (2) using the tensor components of $\varepsilon_{3}$ along main axes: $\varepsilon_{\|}$and $\varepsilon_{\perp}$ [24]. In this Letter, we put $\varepsilon_{\perp}=2, \varepsilon_{\|}=2.91$. Then, $\left(\varepsilon_{\text {eff }}^{\prime}\right)_{\perp} \simeq-0.52$ for $\varepsilon_{3}=\varepsilon_{\perp}$ and $\left(\varepsilon_{\text {eff }}^{\prime}\right)_{\|} \simeq 2$ for $\varepsilon_{3}=\varepsilon_{\|}$. As the real parts of effective permittivities along principal axes are opposite in signs, NDLC is an indefinite medium. The effective permeability is independent on $\varepsilon_{3}$ in the interval of values relevant for this design [Fig. 1(b)], and we put $\left(\mu_{\mathrm{eff}}^{\prime}\right)_{z z} \equiv \mu_{\mathrm{eff}}^{\prime} \simeq 1$.

The tensor components $\left(\varepsilon_{\text {eff }}^{\prime}\right)_{x x}, \quad\left(\varepsilon_{\text {eff }}^{\prime}\right)_{y y},\left(\varepsilon_{\text {eff }}^{\prime}\right)_{x y}$ in coordinates from Fig. 1(a), calculated by rotating the diagonal tensor in principal axes around $z$ axis by angle $\gamma$, are shown in Fig. 2. As angle $\gamma$ characterizes the direction of the optic axis, which can be varied through the external electric field, we conclude that the NDLC becomes (within the limits of validity of the effective medium theory) a tunable hyperbolic medium.

Rotation of the director of NLC accompanied by the variation of the effective permittivity tensor changes the refraction at the boundary isotropic/indefinite medium. In geometric approach this rotation promotes the rotation of the hyperbola of equifrequency contour in NDLC material [14]. In the principal axes of effective permittivity tensor this hyperbola determines the dispersion relation for TM waves in NDLC [2]:

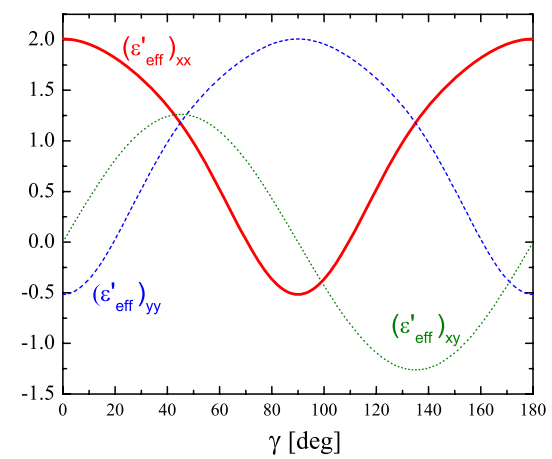

Fig. 2. Dependence of tensor components $\left(\varepsilon_{\text {eff }}^{\prime}\right)_{x x},\left(\varepsilon_{\text {eff }}^{\prime}\right)_{y y}$, and $\left(\varepsilon_{\text {eff }}^{\prime}\right)_{x y}$ on rotation angle $\gamma$.

$$
\frac{k_{x}^{2}}{\left(\varepsilon_{\mathrm{eff}}^{\prime}\right)_{y y}}+\frac{k_{y}^{2}}{\left(\varepsilon_{\mathrm{eff}}^{\prime}\right)_{x x}}=\frac{\omega^{2}}{c^{2}},
$$

where $c$ denotes the velocity of light in vacuum. The case of $\gamma=90^{\circ}$ is shown as an inset in Fig. 3, where the equifrequency contour of an isotropic material is represented by a circle. Based on this diagram, the geometrical analysis of refraction can be done in a standard way [14] by observing the continuity of the tangential components of incident $\left(\vec{k}_{i}\right)$ and refracted $\left(\vec{k}_{r}\right)$ wave vectors. The direction of energy flow described by the Poynting vector $\vec{S}$ within the indefinite medium is indicated by the arrow drawn normal to the hyperbolic isofrequency surface. The refraction angles for the wave beam $\theta_{r, S}$ and for the wave vector can be calculated analytically. The same geometric approach is used in the case of rotated nematic/equifrequency contour (see, e.g., the appendix in [14]).

In the case of uniform configuration of NLC $\left(\gamma=90^{\circ}\right)$, the geometric approach predicts that negative refraction of the TM wave occurs for all angles of incidence $\theta_{i}$ (solid line in Fig. 3). This prediction is confirmed by FE calculations (symbols in Fig. $\underline{3}$ ). Figure $\underline{4}$ characterizes the energy flow and electromagnetic wave in the system for angle of incidence $\theta_{i}=40^{\circ}$. Figure $4(\mathrm{a})$ shows the

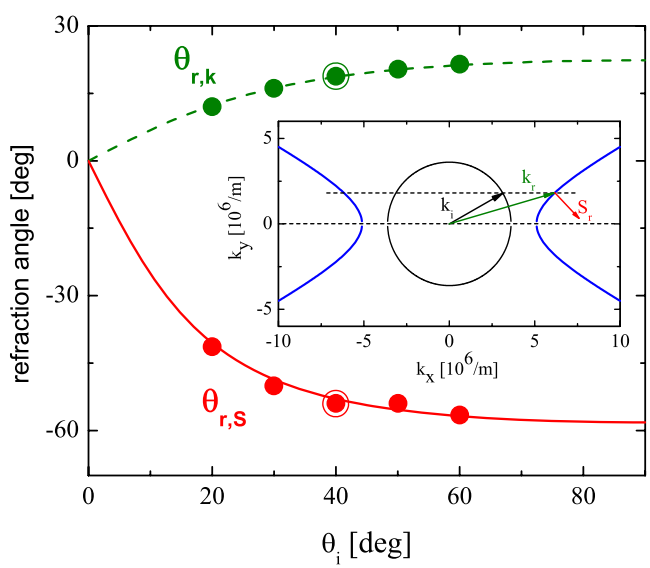

Fig. 3. Plots of refraction angles $\theta_{r, S}\left(\theta_{i}\right)$ (solid line) and $\theta_{r, k}\left(\theta_{i}\right)$ (dashed line) for a uniform configuration $\left(\gamma=90^{\circ}\right)$. Symbols represent FE results [18,19]. Large symbol-see Fig. 4. Inset: isofrequency contours for the TM wave incident from an isotropic material to an indefinite one with $\left(\varepsilon_{\text {eff }}^{\prime}\right)_{x x}<0$ and $\left(\varepsilon_{\text {eff }}^{\prime}\right)_{y y}>0$ for the angle of incidence $\theta_{i}=30^{\circ}$. 


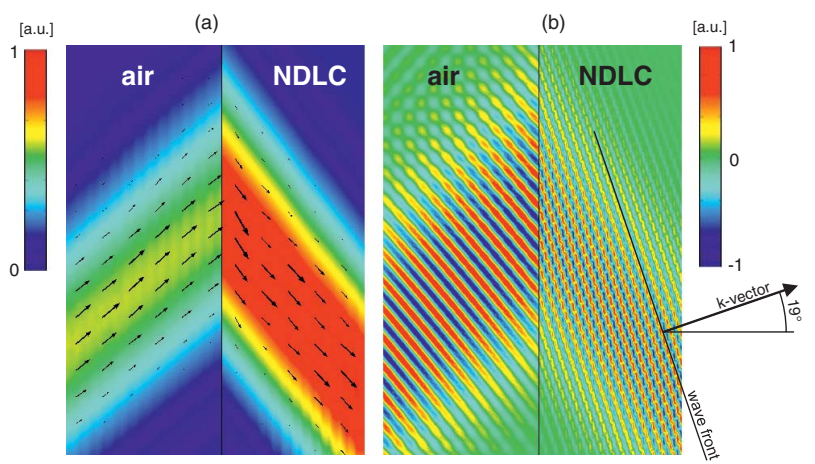

Fig. 4. Finite element simulations of the electric and magnetic field mapping for Gaussian beam with TM polarization, $\theta_{i}=40^{\circ}$. (a) Magnitude of the electric field and Poynting vectors and (b) $z$ component of the magnetic field.

magnitude of the electric field and Poynting vectors, Fig. 4(b) shows the $z$ component of the magnetic field. Direction of vector $\vec{k}_{r}$ for refracted wave and angle of negative refraction for Poynting vector are in agreement with analytical results from Fig. 3 . We point out that using magnetic mapping is a matter of convenience-we have verified that electric mapping yields the same wavefronts as magnetic mapping.

Using the formalism presented above we have studied the behavior of a plane TM wave incident from vacuum on the indefinite NDLC for a few chosen angles of incidence $\theta_{i}$ and for an arbitrary orientation of NLC director $\left(0 \leq \gamma \leq 180^{\circ}\right)$. The dependence of refraction angle $\theta_{r, S}$ on $\gamma$ is shown in Fig. 5. We have singled out three distinct regions. Region II extends from $\gamma=\gamma_{L} \simeq 64^{\circ}$ to $\gamma=\gamma_{U} \approx 116^{\circ}$. Geometric and numeric analysis show that the location of lower boundary $\gamma_{L}$ is independent on $\theta_{i}$ while the location of upper boundary $\gamma_{U}$ is only very weakly dependent on $\theta_{i}$. Refraction can be negative (area below the dashed line $\theta_{r, S}=0$ ) or normal (area above the dashed line $\theta_{r, S}=0$ ), depending on values of $\theta_{i}$ and $\gamma$. For example, for $\theta_{i}=30^{\circ}$ negative refraction is present for $\gamma<106^{\circ}$, positive refraction for $\gamma>106^{\circ}$. For $\theta_{i}>50^{\circ}$ only negative refraction is present for any orientation $\gamma$ of the NLC director in region II. We have used the approach from Ref. [13] to show that in this region the wave vector

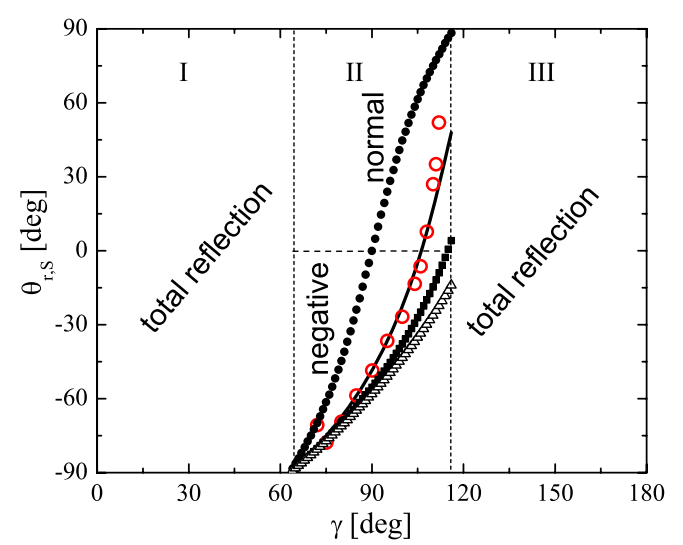

Fig. 5. Refraction angle $\theta_{r, S}$ in function of the orientation $\gamma$ of NLC director for $\theta_{i}=0^{\circ}$ (full circles), $30^{\circ}$ (solid line), $50^{\circ}$ (squares), and $80^{\circ}$ (triangles). Large circles represent FE calculations for $\theta_{i}=30^{\circ}$. of the wave propagating in NDLC HM metamaterial is purely real, which implies that the plane wave impinging from vacuum excites a homogeneous wave. In regions I $\left(\gamma<\gamma_{L}\right)$ and III $\left(\gamma>\gamma_{U}\right)$ the geometric construction breaks down and the wave vector becomes imaginary, which implies that the impinging plane wave excites an evanescent wave.

To verify analytical geometric predictions we have used FE calculations. In regions I and III total reflection occurs (there was no transmitted wave), while in region II the simulations for $\theta_{i}=30^{\circ}$ (symbols) confirm theoretical analysis. Small discrepancies close to boundaries of region II have numerical origin.

These results make possible design of a novel electrooptic effect: an external electric-field-driven real-time switch between negative refraction, positive refraction, and total reflection. To illustrate its functionality we have performed FE simulations of a TM Gaussian beam with $\lambda_{0}=1.74 \mu \mathrm{m}$ impinging a NDLC HM cell with thickness $10 \mu \mathrm{m}$ at angle of incidence $\theta_{i}=30^{\circ}$. In Fig. 6 the magnetic field mapping is shown for three operating
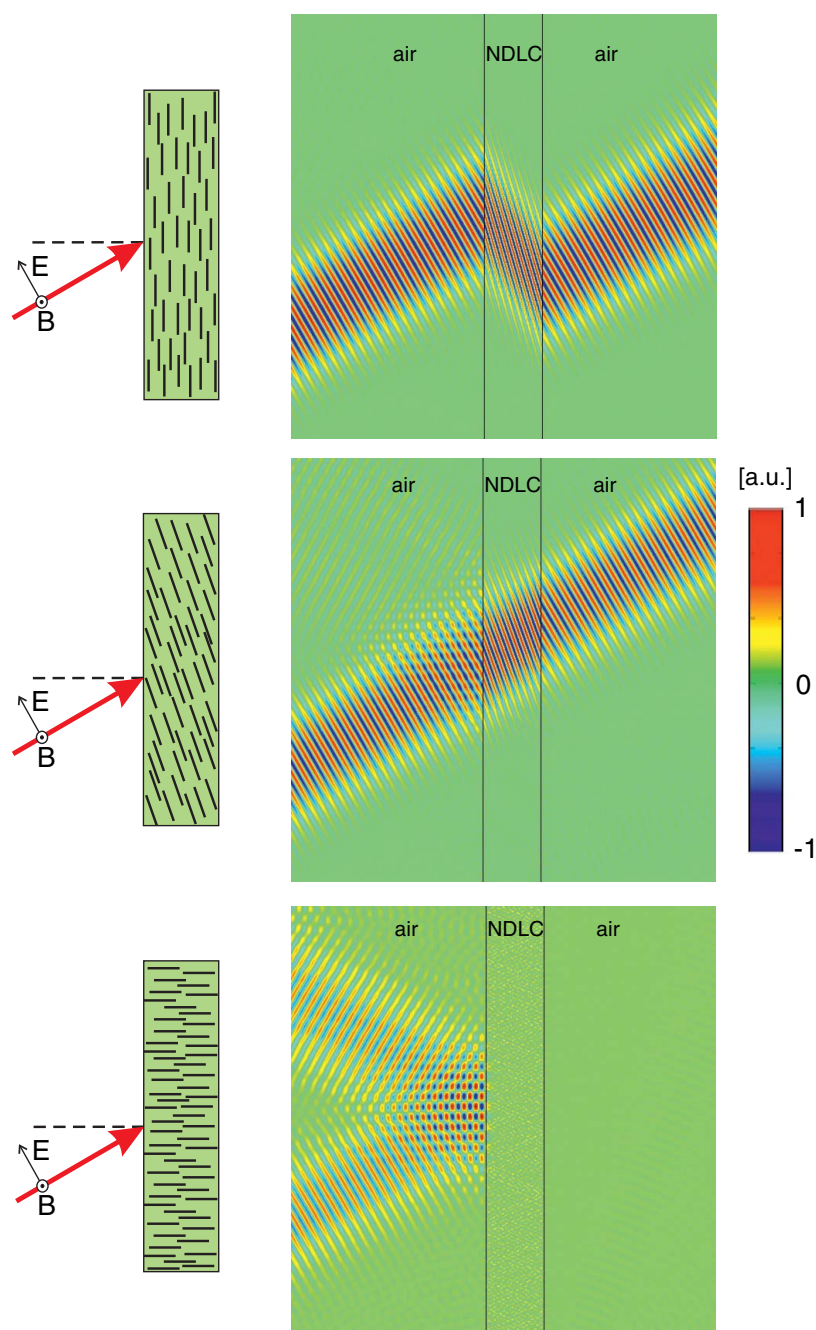

Fig. 6. Operating principle of the switch. Left, orientation of NLC host; right, magnetic field mapping for a Gaussian TM beam incident at $\theta_{i}=30^{\circ}$. Negative refraction $\left(\gamma=90^{\circ}\right.$, top); positive refraction $\left(\gamma=110^{\circ}\right.$, middle); and total reflection $\left(\gamma=0^{\circ}\right.$, bottom $)$. 
states of the switch, which depend on the orientation of the NLC host controlled by an external electric field: negative refraction for $\gamma=90^{\circ}$ (top), positive refraction for $\gamma=110^{\circ}$ (middle), and total reflection for $\gamma=0^{\circ}$ (bottom). External voltage required for a reorientation of NLC amounts to a few volts (Fréedericksz threshold), and the reorientation time varies between several seconds and several milliseconds.

We have shown using an effective medium approach and FE calculations (Comsol) that NDLC metamaterial becomes a tunable indefinite medium in near IR. The tunability is provided by varying the external electric field, which changes the orientation of the NLC director, the overall effective permittivity tensor, and the orientation of the hyperbola of the dispersion relation of extraordinary waves through the medium. The latter allows control of the character of the electromagnetic field in the medium-propagating or evanescent. We have exploited this tunability to design a new electro-optic effect for the manipulation of light-electric-field-driven switch between negative and positive refraction and reflection and have illustrated its functionality. Compared to negative refraction in pure NLC medium [21,22], the NDLC device operates in much wider intervals of incidence/ refraction angles.

This work is supported by the Air Force Office of Scientific Research (AFOSR). G. P. acknowledges the Polish National Science Center for financial support under Grant NN507 322440. K. T. acknowledges support of the Foundation for Polish Science START Program.

\section{References}

1. N. Engheta and R. W. Ziolkowski, Metamaterials: Physics and Engineering Explorations (Wiley, 2006).

2. W. Cai and V. Shalaev, Optical Metamaterials Fundamentals and Applications (Springer, 2010).

3. D. R. Smith and D. Schurig, Phys. Rev. Lett. 90, 077405 (2003).

4. D. R. Smith, P. Kolinko, and D. Schurig, J. Opt. Soc. Am. B 21, 1032 (2004).
5. A. Fang, T. Koschny, and C. M. Soukoulis, Phys. Rev. B 79, 245127 (2009).

6. Z. Jacob, L. V. Alekseyev, and E. Narimanov, Opt. Express 14, 8247 (2006).

7. H. Lee, Z. Liu, Y. Xiong, C. Sun, and X. Zhang, Opt. Express 15, 15886 (2007).

8. J. Yao, K. T. Tsai, Y. Wang, Z. Liu, G. Bartal, Y. L. Wang, and X. Zhang, Opt. Express 17, 22380 (2009).

9. B. D. F. Casse, W. T. Lu, Y. J. Huang, E. Gultepe, L. Menon, and S. Sridhara, Appl. Phys. Lett. 96, 0231114 (2010).

10. J. Yang, X. Hu, X. Li, Z. Liu, X. Jiang, and J. Zi, Opt. Lett. 35, 16 (2010).

11. J. Zhao, Y. Chen, and Y. Fen, Appl. Phys. Lett. 92, 071114 (2008).

12. L. V. Alekseyev, E. E. Narimanov, T. Tumkur, H. Li, Y. A. Barnakov, and M. A. Noginov, Appl. Phys. Lett. 97, 131107 (2010).

13. E. Spinozzi and A. Ciattoni, Opt. Mater. Express 1, 732 (2011).

14. T. M. Grzegorczyk, M. Nikku, X. Chen, B.-I. Wu, and J. A. Kong, IEEE Trans. Microw. Theory Tech. 53, 1443 (2005).

15. S.-H. Liu and L.-X. Guo, Prog. Electromagn. Res. 115, 243 (2011).

16. G. Pawlik, M. Jarema, W. Walasik, A. C. Mitus, and I. C. Khoo, J. Opt. Soc. Am. B 27, 567 (2010).

17. I. C. Khoo, D. H. Werner, X. Liang, A. Diaz, and B. Weiner, Opt. Lett. 31, 2592 (2006).

18. G. Pawlik, W. Walasik, K. Tarnowski, A. C. Mitus, and I. C. Khoo, Proc. SPIE 8828, 88280E (2013).

19. G. Pawlik, W. Walasik, K. Tarnowski, A. C. Mitus, and I. C. Khoo, Proc. SPIE 8901, 890111 (2013).

20. D. Jia, C. Yang, X. Li, Z. Peng, Y. Liu, Z. Cao, Q. Mu, L. Hu, D. Li, L. Yao, X. Lu, X. Xiang, H. Zhang, and L. Xuan, Liq. Cryst. 41, 207 (2014).

21. D. Jia, C. Yang, Z. Peng, X. Li, Y. Liu, L. Yao, Z. Cao, Q. Mu, L. $\mathrm{Hu}, \mathrm{X}$. Lu, and L. Xuan, Liq. Cryst. 40, 599 (2013).

22. O. P. Pishnyak and O. D. Lavrentovich, Appl. Phys. Lett. 89, 251103 (2006).

23. X. Wang, D. H. Kwon, D. H. Werner, I. C. Khoo, A. V. Kildishev, and V. M. Shalaev, Appl. Phys. Lett. 91, 143122 (2007).

24. G. Pawlik, K. Tarnowski, W. Walasik, A. C. Mitus, and I. C. Khoo, Opt. Lett. 37, 1847 (2012). 